\title{
In Vitro Screening of Several Potato Genotypes for Water Stress Using High Agar Levels in the Medium
}

\author{
El-Magawry, N.A. ${ }^{1}$; F.H. Mohamed ${ }^{2}$; K.E. Abdel-Hamid ${ }^{2}$; M.W.M. Elwan ${ }^{2 *}$ and M.M. Abdel-Salam ${ }^{1}$ \\ ${ }^{1}$ Agric. Research Center, Giza, Egypt \\ ${ }^{2}$ Department of Horticulture, Faculty of Agriculture, Suez Canal University, Ismailia, Egypt
}

Received: $22 / 4 / 2015$

\begin{abstract}
In vitro screening of several potato genotypes (27 cultivars and breeding lines) for agar-induced water stress was conducted. Single node explants were evaluated on the basis of their plantlet growth and microtuberization under different concentrations of agar (7-10 g/l). Increasing agar in the tissue culture medium resulted in reduction in plantlet growth, rooting and tuberization potential in varying degrees, depending on the cultivar. Under agar-induced water stress, Safran and Universa were generally among the ranked tolerant cvs; Nicola, Triomph and Agria as moderate; Diamant and Bolista as sensitive to drought. Photosynthetic pigment contents under agar-induced water stress were markedly decreased in most cvs tested. However, the cv. Universa showed larger decline in total chlorophyll than Diamant or Bolista. Biochemical analysis of potato plantlet indicated increase in free amino acids, proline and catalase (CAT) activities under water stress, while superoxide dismutase (SOD) was less than the control. Results also indicated that the free amino acids and CAT activity were positively correlated with the tolerance mechanism to water stress.
\end{abstract}

Keywords: Solanum tuberosum L., abiotic stress, tissue culture, drought.

\section{INTRODUCTION}

Potato (Solanum tuberosum L.) is one of the most important vegetable crops in Egypt for local consumption and exportation. The total area devoted for production in the year 2013 in Egypt was 300,662 Faddan, with total production of about 4,500,000 tons (average 14,96 ton/fad.). Worldwide, potato is the fourth most important crop, with an annual production of 325 million tons, (FAO state; 2012). Abiotic stresses, such as extreme temperature, drought and high salinity often result in significant losses to the yields of economically important crops such as potato (Ahmed and Rashid, 1990). Plants constantly exposed to capricious conditions have adapted at the molecular, cellular, physiological and biochemical levels, enabling them to survive and cope with adverse environmental stresses.

Examining the field performance of potato genotypes under drought stress is the usual method for evaluation, but, the results are often inconclusive. Field trial is normally associated with, non-uniform moisture availability and temperature fluctuations during the growing season. This method involves considerable space, time, labor, equipment and planting material resources (Arvin and Donnelly, 2008). Therefore, in vitro screening of the new currently grown genotypes represents valuable tool as alternative to field trials. Furthermore, a highly significant correlation was found between in vitro growth parameters and field performance of ten potato clones studied by Morpurgo (1991).

Water stress also severally affects potato yield as reported by Singh (1969) and preparing sufficient water is very important for increasing potato quality and quantity. Because of its shallow root system, potatoes are classified as sensitive to drought stress (Yuan et al., 2003). In vitro screening of potato for water stress was carried out with different water stress agents, including PEG (Hassanpanah, 2009; Daneshmand et al., 2010; Pino et al., 2013); sorbitol (Gopal and Kazuto, 2007) and mannitol (Sabbah and Moshe, 1990), using agar levels higher than normal $(7.0 \mathrm{~g} / \mathrm{l})$ was utilized in the study of Gopal et al. (2008). In three potato genotypes, the agar- induced water stress (at $10 \mathrm{~g} / \mathrm{l}$ ) adversely affected plantlet growth and rooting, and the response varied with genotypes. Nistor et al. (2014) used agar in MS medium at 9.0 to $20 \mathrm{~g} / \mathrm{l}$. Leaf and node numbers as well as plantlet length decreased with increasing agar level, depending on the cultivar. Plantlet height and nodes numbers were severely affected and represent about $40 \%$ of the control. Similar results were also found by Shafqatulla et al. (2007). The later reports were only confined to the in vitro response of agarinduced water stress on plantlet growth bioassay but no information about in vitro microtuberization potentials under such condition were available in the literatures. Therefore, the objectives of the present study were to screen large number of potato genotypes for tolerance to water stress using plantlet growth and microtuberization bioassays. Biochemical changes associated with drought tolerance were also determined.

\section{MATERIALS AND METHODS}

The current investigation was conducted at the Plant Tissue Culture Laboratory of the Horticulture Department, Suez Canal University, Ismailia, during the period of 2012 to 2014.

\section{Plant material:}

Twenty seven potato cultivars and breeding lines from different maturity groups, including six early genotypes (Safran, Margod, Universa, Alaska, Spunta and Elodi), six mid-early genotypes (Triomph, Lady Rosetta, Nicola, Fridor, Naga, and Diamant), one late maturing (Agria), in addition to seven potato lines under test (94f-8101, 96f-25-25, 95k-94, 97-980, 94f and 97F267), German varieties (Jelly, Presto and Marabel) and new locally-grown cultivars (Picasso, Proventa, Arinda, 
Bolista and Sante) were used for the study. These potato genotype collections were continuously micropropagated and kept as plantlet grown in test tubes under $20 \pm 2^{\circ} \mathrm{C}$ in our tissue culture facility at the Department of Horticulture, Faculty of Agriculture, Suez Canal University.

\section{Experimental protocol:}

Under sterile conditions, 10 nodes (1 cm long) were cultured per jar containing $30 \mathrm{ml}$ MS medium (Murashige and Skoog, 1962) amended with the agar treatments $(7,8,9$ and $10 \mathrm{~g} / \mathrm{l})$. After 6 weeks, 5 plantlets were taken from each cultivar $\mathrm{x}$ agar treatment and data were taken on plantlet length and root number/plantlet.

To test in vitro microtuberization under the previous agar treatments, $30 \mathrm{ml}$ sterilized liquid MS medium amended with high sucrose level $(80 \mathrm{~g} / \mathrm{l})$ were added to each jar containing the growing plantlets after removal of the jar cap in a laminar air-flow hood. Cultures were incubated in the dark at $18-20^{\circ} \mathrm{C}$ for 2 months. Microtubers produced from each treatment were harvested and data were taken on number and weight (yield) of microtuber/jar and the average single microtuber weight were calculated by dividing weight/number. Percent of tuber formation under the highest agar level (10 g/l) was also calculated as relative to the control.

\section{Biochemical analysis of potato plant grown under in vitro water stress conditions.}

Based on the morphological characters (plantlet height) only seven potato genotypes were chosen for biochemical analysis as representing, 1) tolerant genotypes (Universa and Safran). 2) moderately tolerant genotypes (cvs. Triomph, Nicola and Agria), and 3) sensitive genotypes (Diamant and Bolista). The biochemical analysis was made only in plantlets exposed to highest agar concentration $(10 \mathrm{~g} / \mathrm{l})$ in comparison with control plantlets.

1-Chlorophyll and carotenoid contents were determined according to the method of Lichtenthaler (1987).

2- Free total amino acids were colorimetrically assayed by ninhydrin reagent at $570 \mathrm{~nm}$ according to the method described by Lee and Takabashi (1966).

3-Proline was estimated using the method described by Sadasivam and Manickam (1991).

4- Enzymes: The plantlet samples (weight 0.1-0.4g) were prepared for enzyme activity as described by $\mathrm{Ni}$ et al. (2001).

4-a. Superoxide dismutase (SOD) activity was determined by spectrophotometriclly at $560 \mathrm{~nm}$ (U\V Spectrophotometer spectronic 1201, Milton Roy, U.S.A) as described by the method of Nishikimi et al. (1972).

4-b. Catalase (CAT) activity was measured spectrophotometriclly at $510 \mathrm{~nm}$ as described by the method of Aebi (1984).

\section{Statistical analysis:}

The experimental design was a randomized complete block design in factorial fashion. Data were combined and subjected to ANOVA using CoStat program and the means were separated by DMRT test at $5 \%$ level.

\section{RESULTS}

In this experiment, 27 potato genotypes were screened for their shoot and root growth and microtuberization in vitro under different agar concentrations (7.0 g/l as control, 8.0, 9.0 and $10.0 \mathrm{~g} / \mathrm{l})$ in the tissue culture medium specific for shoot growth or microtuber formation.

\section{a. Effect of agar and genotypes on plantlet growth: \\ a. 1. Main effects of agar:}

Results revealed that the differences among agar concentrations as averaged over genotypes, were significant. Moderate $(8.0 \mathrm{~g} / \mathrm{l})$ agar level in the medium recorded the highest plantlet length (Table 1) over the control and agar at $9.0 \mathrm{~g} / \mathrm{l}$ which were not significantly different. Plantlet length at the highest agar $(10.0 \mathrm{~g} / \mathrm{l})$ was significantly the least (ave. $2.93 \mathrm{~cm}$ ), representing $48.3 \%$ of the control. Root number per plantlet was significantly affected by agar concentration (Table 2). Averaged over all genotypes, root number at $8.0 \mathrm{~g} / \mathrm{l}$ agar recorded the highest value (ave. 4.86 roots) compared to the control (ave. 2.97 roots) and agar at $9.0 \mathrm{~g} / 1$ (ave. 2.64 roots), while the least average root number was found in plantlet grown at $10.0 \mathrm{~g} / \mathrm{l}$ agar (ave. 0.52 root) representing only $17.5 \%$ of the control and $10.7 \%$ of the root number recorded at $8.0 \mathrm{~g} / \mathrm{l}$ agar. Therefore, rooting of potato plantlet was more affected by agar - induced water stress than plantlet length.

\section{a. 2. Main effects of genotypes:}

As an average over the tested agar concentrations, potato genotypes were found to be significantly different in shoot and root characters. Plantlet length in cvs. Marabel, 95k-94 and Diamant recorded the highest values (ave. 7.1-8.2 cm) followed by cvs. Agria, Presto and Proventa. The least plantlet height was observed on cvs. Alaska, 97-980 and Arinda (about $3.3 \mathrm{~cm}$ ) as shown in Table (1).

Number of roots/plantlet was significantly different among the potato genotypes in this experiment (Table $2)$. As tested over agar levels, the cv. Alaska recorded the highest number of roots/plantlet (ave. 6.4 roots), followed by cvs. Universa (ave. 5.95 roots), cv. Safran (ave. 5.45 roots), 95k-94 and Diamant (ave. 5.2 roots/plantlet).

\section{a. 3. Effect of Agar $x$ genotype interaction:}

Results indicated that shoot length was significantly affected by the agar $x$ genotype interactions (Table 1). At $7.0 \mathrm{~g} / \mathrm{l}$, the cvs Marabel, Presto and Diamant, followed by $95 \mathrm{k}-94,96 \mathrm{~F}-25-25$ and Safran recorded the highest plantlet length $(8.2-10.33 \mathrm{~cm})$, while at $8.0 \mathrm{~g} / \mathrm{l}$, the cvs. Agria and Marbel had the highest shoot length among all agar $x$ genotype combinations $(>10.0 \mathrm{~cm})$, and was followed by cvs. 95k-94, Presto and Proventa. On the other hand, the cvs.Safran and Marabel had the highest shoot length at $9.0 \mathrm{~g} / \mathrm{l}$ agar. None of the tested genotypes had shoot length higher than the control when grown on medium with high agar level $(10.0 \mathrm{~g} / \mathrm{l})$. The interaction of agar $\mathrm{x}$ genotype was found significant for number of roots/plantlet (Table 2). While the highest root number was found on cv. Diamant under $7.0 \mathrm{~g} / \mathrm{l}$ agar (ave. 10.0 roots/plantlet), the highest rooting of 
cvs. Marabel and Picasso was at 8.0g/1 agar (ave. 9.09.3 roots). Most cvs. ( $22 \mathrm{cvs}$.) did not form roots at the highest agar-induced water stress level $(10.0 \mathrm{~g} / \mathrm{l})$.

b. Effects of agar levels and genotypes on microtuberization:

Results revealed significant main effects of agar and potato genotypes, as well as their interaction on several aspects of potato microtuberization.

b.1. Main effects of agar concentrations on microtuberization:

Agar levels on the medium significantly affected thenumber of microtuber produced/jar (Table 3). As an average over all genotypes, there were no significant differences in microtuber number between 7.0 and $8.0 \mathrm{~g} / \mathrm{l}$ (ave. 5.3 and 4.9 microtuber/jar). At 9.0 and $10.0 \mathrm{~g} / \mathrm{l}$ agar, microtuber number decreased (ave 3.6 and 1.97, respectively). At $10.0 \mathrm{~g} / \mathrm{l}$ agar, the number of microtuber was only $36.8 \%$ of the control. The average microtuber weight was also affected by the agar concentrations, which was decreased dramatically with the increase in agar level in the medium (Table 4). The highest average microtuber weight was recorded at the control agar treatment $(7.0 \mathrm{~g} / \mathrm{l})$ reaching ave. 519 $\mathrm{mg} /$ tuber, followed by agar at $8.0 \mathrm{~g} / 1$ (432 mg), $9.0 \mathrm{~g} / \mathrm{l}$ $(284 \mathrm{mg})$, and was the least at the highest agar level (ave. $72.0 \mathrm{mg}$ ), representing only $13.8 \%$ of the control at $7.0 \mathrm{~g} / 1$ agar.

Table (1). Effect of agar concentration on the in vitro shoot (plantlet) length in 27 potato genotypes.

\begin{tabular}{|c|c|c|c|c|c|c|c|c|c|c|c|}
\hline \multirow{3}{*}{ Genotype } & \multicolumn{8}{|c|}{ Agar concentration (g/l) } & & & \multirow{3}{*}{$\begin{array}{c}\% \text { of } \\
\text { control** }\end{array}$} \\
\hline & 7 & & 8 & & 9 & & 10 & & \multicolumn{2}{|c|}{ Mean Genotype } & \\
\hline & \multicolumn{8}{|c|}{ Plantlet length (cm) } & & & \\
\hline Safran & $3.450^{*}$ & E-M & 7.950 & $e-i$ & 9.400 & $a-d$ & 2.550 & K-S & 5.838 & efg & 73.913 \\
\hline $94 f-8101$ & 8.200 & d-h & 7.300 & $g-n$ & 2.900 & H-Q & 5.250 & s-A & 5.913 & ef & 64.024 \\
\hline Margod & 3.500 & E-M & 3.620 & D-L & 5.200 & $\mathrm{~s}-\mathrm{A}$ & 3.100 & H-P & 3.855 & $\mathrm{klm}$ & 88.571 \\
\hline Universa & 5.200 & $\mathrm{~s}-\mathrm{A}$ & 8.700 & $c-f$ & 3.800 & B-K & 3.550 & F-N & 5.250 & gh & 68.269 \\
\hline Alaska & 2.850 & H-Q & 5.250 & s-A & 3.850 & C-J & 1.800 & Q-U & 3.438 & $\mathrm{mn}$ & 63.158 \\
\hline Spunta & 6.850 & $\mathrm{i}-\mathrm{q}$ & 6.600 & j-r & 6.250 & $1-\mathrm{t}$ & 3.200 & $\mathrm{G}-\mathrm{O}$ & 5.725 & efg & 46.715 \\
\hline Elodi & 3.850 & B-J & 7.550 & $\mathrm{f}-\mathrm{k}$ & 4.850 & $\mathrm{v}-\mathrm{D}$ & 1.950 & $\mathrm{O}-\mathrm{U}$ & 4.550 & $\mathrm{ij}$ & 50.649 \\
\hline $96 f-25-25$ & 8.700 & $c-f$ & 7.150 & g-o & 5.800 & $\mathrm{p}-\mathrm{x}$ & 1.540 & $\mathrm{R}-\mathrm{U}$ & 5.798 & efg & 17.701 \\
\hline Triomph & 4.050 & A-I & 6.900 & $i-p$ & 5.070 & $t-B$ & 2.100 & $\mathrm{~N}-\mathrm{U}$ & 4.530 & $\mathrm{ij}$ & 51.852 \\
\hline Lady Rosetta & 4.900 & $\mathrm{u}-\mathrm{C}$ & 3.850 & B-J & 4.600 & $x-E$ & 1.300 & STU & 3.663 & $\operatorname{lm}$ & 26.531 \\
\hline 95k-94 & 8.950 & b-e & 8.950 & $b-e$ & 8.400 & $d-g$ & 3.050 & H-Q & 7.338 & A & 34.078 \\
\hline Nicola & 6.300 & $\mathrm{k}-\mathrm{t}$ & 6.450 & $\mathrm{k}-\mathrm{s}$ & 5.150 & $\mathrm{t}-\mathrm{A}$ & 3.200 & $\mathrm{G}-\mathrm{O}$ & 5.275 & fgh & 50.794 \\
\hline Fridor & 5.750 & $\mathrm{p}-\mathrm{x}$ & 6.600 & $\mathrm{j}-\mathrm{r}$ & 5.950 & o-w & 4.720 & w-E & 5.755 & efg & 82.087 \\
\hline Naga & 5.700 & $\mathrm{p}-\mathrm{y}$ & 6.900 & i-p & 5.960 & o-w & 1.250 & $\mathrm{TU}$ & 4.953 & hi & 21.930 \\
\hline Diamant & 9.700 & $a b c$ & 7.450 & f-1 & 7.300 & g-n & 4.050 & A-I & 7.125 & $\mathrm{ab}$ & 41.753 \\
\hline Agria & 7.400 & g-m & 10.350 & $\mathrm{a}$ & 5.500 & $r-z$ & 3.750 & $\mathrm{C}-\mathrm{K}$ & 6.750 & $a b c$ & 50.676 \\
\hline 97-980 & 3.300 & F-N & 4.450 & $y-G$ & 4.090 & A-H & 2.100 & $\mathrm{~N}-\mathrm{U}$ & 3.485 & $\mathrm{mn}$ & 63.636 \\
\hline $94 f$ & 7.850 & $e-j$ & 7.750 & $e-j$ & 4.400 & $\mathrm{z}-\mathrm{G}$ & 3.500 & E-M & 5.875 & efg & 44.586 \\
\hline Jelly & 5.050 & $t-B$ & 6.100 & $\mathrm{n}-\mathrm{v}$ & 3.100 & H-P & 3.250 & $\mathrm{G}-\mathrm{N}$ & 4.375 & ijk & 64.356 \\
\hline Presto & 9.700 & $a b c$ & 8.350 & d-g & 7.400 & $\mathrm{~g}-\mathrm{m}$ & 1.000 & $\mathrm{U}$ & 6.613 & bcd & 10.309 \\
\hline Marabel & 10.330 & $\mathrm{a}$ & 10.050 & $a b$ & 9.700 & $a b c$ & 2.800 & I-R & 8.220 & cde & 27.106 \\
\hline $97 f-267$ & 5.250 & $\mathrm{~s}-\mathrm{A}$ & 6.950 & h-p & 1.850 & $\mathrm{P}-\mathrm{U}$ & 1.550 & $\mathrm{R}-\mathrm{U}$ & 3.900 & $\mathrm{n}$ & 29.524 \\
\hline Picasso & 7.300 & g-n & 5.600 & $\mathrm{q}-\mathrm{z}$ & 5.750 & $\mathrm{p}-\mathrm{x}$ & 4.550 & $x-F$ & 5.800 & efg & 62.329 \\
\hline Proventa & 4.070 & A-I & 6.900 & $i-p$ & 7.850 & $e-j$ & 5.900 & $\mathrm{p}-\mathrm{w}$ & 6.180 & cde & 144.963 \\
\hline Arinda & 3.300 & $\mathrm{~F}-\mathrm{N}$ & 5.300 & s-A & 2.400 & L-T & 2.330 & M-T & 3.333 & $\mathrm{mn}$ & 70.606 \\
\hline Bolista & 7.850 & $e-j$ & 6.150 & $\mathrm{~m}-\mathrm{u}$ & 7.550 & $f-k$ & 2.700 & $\mathrm{~J}-\mathrm{R}$ & 6.063 & de & 34.395 \\
\hline Sante & 5.450 & $r-z$ & 5.550 & $r-z$ & 2.900 & H-Q & 3.300 & F-N & 4.300 & $\mathrm{jkl}$ & 60.550 \\
\hline Mean Agar & 6.088 & $\mathrm{a}$ & 6.841 & $\mathrm{a}$ & 5.443 & $\mathrm{~b}$ & 2.938 & $\mathrm{c}$ & & & \\
\hline
\end{tabular}

* Means with the same letters are not significantly different at $\mathrm{p} \leq 5 \%$.

** Value for each cv. at $10.0 \mathrm{~g} / \mathrm{l}$ agar as relative to the control. 
Table (2). Effect of agar concentration on root number per plantlets in 27 potato genotypes.

\begin{tabular}{|c|c|c|c|c|c|c|c|c|c|c|c|}
\hline \multirow{4}{*}{$\begin{array}{l}\text { Genotype } \\
\text { Safran }\end{array}$} & \multicolumn{10}{|c|}{ Agar concentration (g/l) } & \multirow{4}{*}{$\begin{array}{c}\begin{array}{c}\text { \% of } \\
\text { control }^{* *}\end{array} \\
22.89\end{array}$} \\
\hline & \multicolumn{2}{|c|}{7} & \multicolumn{2}{|c|}{8} & \multicolumn{2}{|c|}{9} & \multirow{2}{*}{\multicolumn{2}{|c|}{10}} & \multirow{2}{*}{\multicolumn{2}{|c|}{$\begin{array}{c}\text { Mean } \\
\text { Genotype }\end{array}$}} & \\
\hline & \multicolumn{6}{|c|}{ Root no. per plantlets } & & & & & \\
\hline & $8.300^{*}$ & $b-e$ & 8.000 & $c-f$ & 3.600 & $\mathrm{p}-\mathrm{u}$ & 1.900 & $x-D$ & 5.450 & $\mathrm{bc}$ & \\
\hline $94 f-8101$ & 1.000 & $\mathrm{D}-\mathrm{G}$ & 2.200 & $\mathrm{w}-\mathrm{C}$ & 2.700 & $\mathrm{u}-\mathrm{A}$ & 0.000 & $\mathrm{G}$ & 1.475 & ijkl & 0.000 \\
\hline Margod & 1.600 & A-E & 7.600 & d-g & 3.900 & $o-t$ & 0.000 & G & 3.275 & e & 0.000 \\
\hline Universa & 2.400 & $v-B$ & 8.500 & bcd & 6.300 & h-k & 6.600 & $g-j$ & 5.950 & $a b$ & 275.0 \\
\hline Alaska & 8.800 & $\mathrm{bc}$ & 8.000 & $c-f$ & 8.800 & bc & 0.000 & G & 6.400 & $\mathrm{a}$ & 0.000 \\
\hline Spunta & 3.100 & s-w & 1.700 & $\mathrm{z}-\mathrm{E}$ & 3.300 & $\mathrm{r}-\mathrm{w}$ & 0.000 & G & 2.025 & fghi & 0.000 \\
\hline Elodi & 1.000 & D-G & 8.000 & $c-f$ & 1.400 & B-E & 0.000 & $\mathrm{G}$ & 2.600 & fgh & 0.000 \\
\hline $96 f-25-25$ & 1.300 & B-F & 1.900 & $x-D$ & 0.000 & $\mathrm{G}$ & 0.000 & G & 0.800 & $\mathrm{mn}$ & 0.000 \\
\hline Triomph & 1.700 & $z-E$ & 5.900 & $\mathrm{i}-1$ & 0.000 & G & 0.000 & G & 1.900 & ghi & 0.000 \\
\hline Lady Rosetta & 2.900 & s-y & 4.300 & $n-r$ & 2.300 & $\mathrm{w}-\mathrm{C}$ & 0.000 & G & 2.375 & fgh & 0.000 \\
\hline 95k-94 & 5.500 & $j-m$ & 6.800 & ghi & 8.800 & $\mathrm{bc}$ & 0.000 & $\mathrm{G}$ & 5.275 & $\mathrm{c}$ & 0.000 \\
\hline Nicola & 1.800 & $y-D$ & 5.400 & $\mathrm{k}-\mathrm{n}$ & 2.900 & $s-y$ & 0.000 & G & 2.525 & fg & 0.000 \\
\hline Fridor & 5.200 & $k-n$ & 2.400 & $v-B$ & 2.900 & s-y & 0.000 & G & 2.625 & $\mathrm{f}$ & 0.000 \\
\hline Naga & 1.900 & $\mathrm{x}-\mathrm{D}$ & 4.600 & $\mathrm{~m}-\mathrm{q}$ & 1.800 & $y-D$ & 0.000 & G & 2.075 & fghi & 0.000 \\
\hline Diamant & 10.000 & $\mathrm{a}$ & 6.600 & $g-j$ & 4.000 & o-s & 0.200 & $\mathrm{FG}$ & 5.200 & $\mathrm{c}$ & 2.000 \\
\hline Agria & 0.900 & D-G & 3.800 & $\mathrm{o}-\mathrm{u}$ & 2.400 & $\mathrm{v}-\mathrm{B}$ & 0.000 & G & 1.775 & hij & 0.000 \\
\hline $97-980$ & 1.000 & D-G & 4.700 & $m-p$ & 0.000 & G & 0.000 & $\mathrm{G}$ & 1.425 & $\mathrm{jklm}$ & 0.000 \\
\hline $94 f$ & 3.700 & $\mathrm{o}-\mathrm{u}$ & 2.400 & $v-B$ & 0.000 & $\mathrm{G}$ & 0.000 & $\mathrm{G}$ & 1.525 & ijk & 0.000 \\
\hline Jelly & 1.200 & $\mathrm{C}-\mathrm{F}$ & 2.300 & w-C & 0.000 & G & 0.000 & G & 0.875 & $\operatorname{lmn}$ & 0.000 \\
\hline Presto & 7.000 & $f-i$ & 0.000 & $\mathrm{G}$ & 0.000 & $\mathrm{G}$ & 0.000 & $\mathrm{G}$ & 1.750 & hij & 0.000 \\
\hline Marabel & 1.000 & D-G & 9.000 & $a b c$ & 4.800 & 1-o & 0.000 & G & 3.700 & de & 0.000 \\
\hline $97 f-267$ & 1.000 & D-G & 4.800 & $1-0$ & 0.000 & G & 0.000 & G & 1.450 & $\mathrm{jklm}$ & 0.000 \\
\hline Picasso & 1.600 & A-E & 9.300 & $a b$ & 7.000 & $f-i$ & 2.400 & $v-B$ & 5.075 & $\mathrm{c}$ & 150.0 \\
\hline Proventa & 3.500 & $q-v$ & 7.200 & e-h & 2.200 & $\mathrm{w}-\mathrm{C}$ & 3.000 & $s-x$ & 3.975 & d & 85.71 \\
\hline Arinda & 1.000 & D-G & 1.000 & D-G & 0.000 & G & 0.000 & G & 0.667 & $\mathrm{n}$ & 0.000 \\
\hline Bolista & 0.600 & EFG & 2.800 & $t-z$ & 2.400 & v-B & 0.000 & $\mathrm{G}$ & 1.450 & ijkl & 0.000 \\
\hline Sante & 1.700 & $z-E$ & 1.900 & $x-D$ & 0.000 & G & 0.000 & G & 0.900 & $\mathrm{klm}$ & 0.000 \\
\hline Mean Agar & 2.982 & $\mathrm{~b}$ & 4.856 & $\mathrm{a}$ & 2.648 & bcd & 0.522 & $\mathrm{c}$ & & & \\
\hline
\end{tabular}

* Means with the same letters are not significantly different at $\mathrm{p} \leq 5 \%$.

** Value for each $\mathrm{cv}$. at $10.0 \mathrm{~g} / \mathrm{l}$ agar as relative to the control.

\section{b.2. Main effect of genotypes on microtuberization:}

Significant differences among potato genotypes were observed on number of microtuber formed/jar (Table 3). The cvs. Safran and Presto had the highest average microtuber number/jar as an average over the tested agar levels, reaching an average of 8.0 microtubers/jar, followed by cvs. Fridor and Naga (ave. 7.3 microtuber/jar), 95k-94 and 94f-8101 (6.5 microtubers). Genotypes were significantly different in microtuber weight (Table 4). The cvs. Safran and 95k94 recorded the highest ave. microtuber weight $(893$ $\mathrm{mg} /$ tuber and $853 \mathrm{mg} /$ tuber), followed by $\mathrm{cv}$. Presto (733mg), cv. Nicola (694mg) and cv. 94f.8101 $(622.0 \mathrm{mg})$. The least microtuber weight was detected in cvs. Picasso, 97f-267, Sante, 97-980, Agria and 96f-2525.

\section{b.3. Effect of agar $x$ genotype interaction on microtuberization:}

Number of microtuber per jar was significantly affected by the interaction of agar $\mathrm{x}$ genotype (Table 3 ).
The cv. Safran recorded the highest number of microtuber produced per jar (ave. 12.67 microtubers) on medium supplemented with $8.0 \mathrm{~g} / \mathrm{l}$ agar. Also, the cvs 95k-94 and Safran had the highest microtuber number (ave. 10.0 microtubers/jar) at 7.0g/l. At higher agar level $(9.0 \mathrm{~g} / \mathrm{l})$ the $\mathrm{cv}$. Presto recorded higher microtuber number (ave. 12 microtubers) among the rest of cvs. under this agar level. At the highest agar level, the cvs. Naga and Fridor were the highest in microtuberization under water stress.

The average microtuber weight was significantly affected by the interaction of agar $x$ genotype (Table 4). On medium with $7.0 \mathrm{~g} / \mathrm{l}$ agar, the cv. Safran and $95 \mathrm{k}-94$ recorded the highest microtuber weight $(>1.8 \mathrm{~g} /$ tuber). The cv. Safran also had higher microtuber weight at $8.0 \mathrm{~g} / \mathrm{l}$ agar, while the cv. Presto recorded the highest microtuber weight at $9.0 \mathrm{~g} / \mathrm{l}$ agar (ave. $1.48 \mathrm{~g} /$ tuber). At the highest agar $(10.0 \mathrm{~g} / \mathrm{l})$ treatment, the $\mathrm{cv}$. Fridor had more microtuber weight $(624 \mathrm{mg} /$ tuber $)$ than all cvs under this agar level. 
Table (3).Effect of agar concentration on the in vitro average microtuber number/jar in 27 potato genotypes.

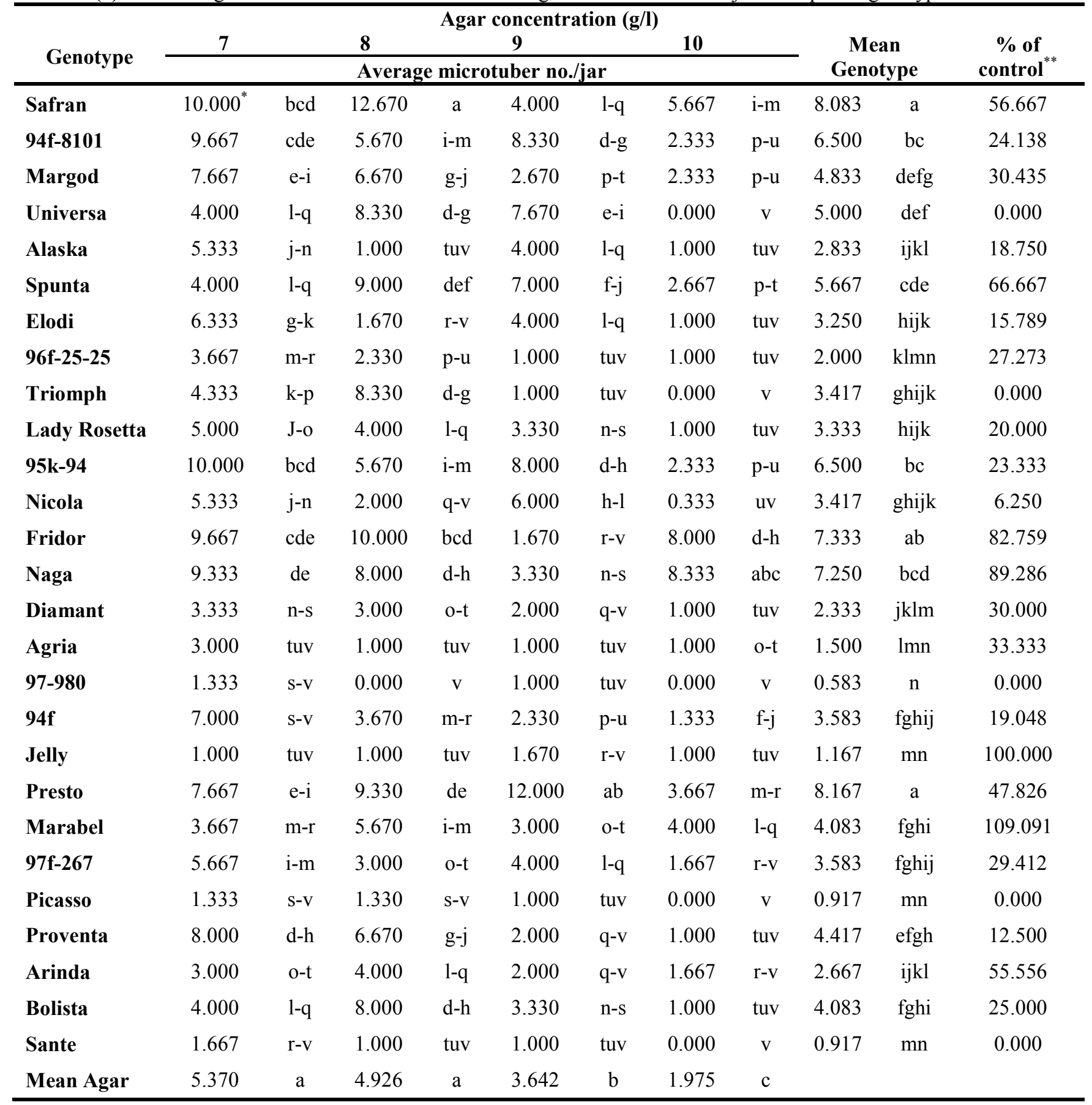

* Means with the same letters are not significantly different at $\mathrm{p} \leq 5 \%$.

** Value for each cv. at $10.0 \mathrm{~g} / \mathrm{l}$ agar as relative to the control.

c. Screening results of potato genotypes for in vitro water stress using high agar concentration in the tissue culture medium:

The main effects of agar-induced water stress indicated its significant effect on plantlet growth, rooting and microtuber induction and development in vitro. Classification of the 27 potato cultivars and new breeding lines into tolerant, moderate and sensitive groups were made taking the measure of growth and development values under the highest used agar level $(10.0 \mathrm{~g} / \mathrm{l})$ as percent of control. Based on plantlet growth at $10 \mathrm{~g} / \mathrm{l}$ agar as $\%$ of control (Table 1), potato genotypes are ranked as follow:
Proventa $>$ Margod $>$ Fridor $>$ Safran $>$ Arinda $>$ Universa $>9$ 4F-8101 $>$ Jelly $>$ 99-981 $>$ Alaska $>97-980>$ Picasso

$>$ Sante $>$ Triomph $>$ Elodi $=$ Agria $=$ Nicola $>$ Spunta $>$ 94F $>$ Diamant $>95 \mathrm{~K}-94>$ Bolista $>$ 97F-267 $>$ Marabel $>$ Lady Rosetta $>$ Naga $>96$ F $-25-25>$ Presto.

From Table (3), based on microtuber number under high agar level as \% of control, the potato genotypes could be ranked as follow: Marabel $>$ Jelly $>$ Naga $>$ Fridor $>$ Spunta $>$ Safran $>$ Arinda $>$

Presto $>$ Agria $>$ Margod $=$ Diamant $>$ 97F-267 $>$ 96F-25$25>$ Bolista $>$ 94F-8101 > 95K-94 > Lady Rosetta $>$ 94F $>$ Alaska $>$ Elodi $>$ Proventa $>$ Nicola $>$ Universa $=$ Triomph $=$ Picasso $=$ Sante $=97-980$. 
Table (4). Effect of agar concentration on the in vitro average microtuber weight in 27 potato genotypes.

\begin{tabular}{|c|c|c|c|c|c|c|c|c|c|c|c|}
\hline \multicolumn{12}{|c|}{ Agar concentration (g/l) } \\
\hline \multirow{3}{*}{$\begin{array}{l}\text { Genotype } \\
\text { Safran }\end{array}$} & \multicolumn{2}{|l|}{7} & \multirow{2}{*}{$\frac{8}{\text { Average }}$} & \multicolumn{2}{|c|}{9} & \multicolumn{3}{|c|}{10} & \multirow{2}{*}{\multicolumn{2}{|c|}{ Mean Genotype }} & \multirow{3}{*}{$\begin{array}{c}\begin{array}{c}\text { \% of } \\
\text { control }^{* *}\end{array} \\
13.472\end{array}$} \\
\hline & \multicolumn{7}{|c|}{ Average microtuber weight (g) } & & & & \\
\hline & $1.874^{*}$ & $\bar{a}$ & 1.230 & $\overline{b c}$ & 0.210 & $\mathrm{t}-\mathrm{C}$ & 0.253 & $\mathrm{~s}-\mathrm{C}$ & 0.893 & $\mathrm{a}$ & \\
\hline 94f-8101 & 1.072 & $\mathrm{~cd}$ & 0.650 & $\mathrm{f}-1$ & 0.760 & e-i & 0.007 & $\mathrm{C}$ & 0.622 & cde & 0.606 \\
\hline Margod & 0.549 & $\mathrm{i}-\mathrm{q}$ & 0.950 & de & 0.320 & $n-y$ & 0.269 & $\mathrm{r}-\mathrm{C}$ & 0.523 & ef & 49.013 \\
\hline Universa & 0.369 & $\mathrm{~m}-\mathrm{x}$ & 0.400 & $1-\mathrm{v}$ & 0.170 & $\mathrm{v}-\mathrm{C}$ & 0.000 & $\mathrm{C}$ & 0.234 & hijk & 0.000 \\
\hline Alaska & 0.752 & $e-i$ & 0.050 & $\mathrm{z}-\mathrm{C}$ & 0.570 & h-p & 0.078 & $\mathrm{y}-\mathrm{C}$ & 0.363 & gh & 10.376 \\
\hline Spunta & 0.369 & $\mathrm{~m}-\mathrm{x}$ & 0.400 & $1-\mathrm{v}$ & 0.260 & $\mathrm{r}-\mathrm{C}$ & 0.031 & $\mathrm{ABC}$ & 0.266 & hijk & 8.327 \\
\hline Elodi & 0.775 & $e-i$ & 0.860 & d-g & 0.610 & $g-m$ & 0.004 & $\mathrm{C}$ & 0.564 & def & 0.478 \\
\hline $96 f-25-25$ & 0.063 & $\mathrm{y}-\mathrm{C}$ & 0.040 & $\mathrm{z}-\mathrm{C}$ & 0.030 & $\mathrm{BC}$ & 0.019 & $\mathrm{BC}$ & 0.037 & $\mathrm{mn}$ & 30.466 \\
\hline Triomph & 0.175 & $\mathrm{v}-\mathrm{C}$ & 0.920 & def & 0.230 & $\mathrm{t}-\mathrm{C}$ & 0.000 & $\mathrm{C}$ & 0.330 & hi & 0.000 \\
\hline $\begin{array}{l}\text { Lady } \\
\text { Rosetta }\end{array}$ & 0.960 & de & 0.380 & $1-x$ & 0.080 & $y-C$ & 0.017 & $\mathrm{BC}$ & 0.359 & gh & 1.772 \\
\hline 95k-94 & 1.929 & $\mathrm{a}$ & 0.510 & i-s & 0.940 & de & 0.036 & $\mathrm{z}-\mathrm{C}$ & 0.853 & $a b$ & 1.889 \\
\hline Nicola & 1.080 & $\mathrm{~cd}$ & 0.960 & de & 0.710 & e-k & 0.029 & $\mathrm{ABC}$ & 0.694 & $\mathrm{~cd}$ & 2.692 \\
\hline Fridor & 0.303 & $\mathrm{p}-\mathrm{z}$ & 0.390 & $1-w$ & 0.030 & $\mathrm{BC}$ & 0.624 & $g-m$ & 0.335 & hi & 206.357 \\
\hline Naga & 0.083 & $\mathrm{y}-\mathrm{C}$ & 0.450 & $\mathrm{k}-\mathrm{u}$ & 0.150 & $\mathrm{v}-\mathrm{C}$ & 0.070 & $\mathrm{y}-\mathrm{C}$ & 0.189 & $\mathrm{jkl}$ & 84.259 \\
\hline Diamant & 0.730 & $e-j$ & 0.390 & $1-w$ & 0.050 & $\mathrm{z}-\mathrm{C}$ & 0.035 & $\mathrm{z}-\mathrm{C}$ & 0.300 & hij & 4.728 \\
\hline Agria & 0.035 & $\mathrm{z}-\mathrm{C}$ & 0.090 & $\mathrm{y}-\mathrm{C}$ & 0.020 & $\mathrm{BC}$ & 0.007 & $\mathrm{C}$ & 0.038 & $\mathrm{mn}$ & 20.000 \\
\hline 97-980 & 0.044 & $\mathrm{z}-\mathrm{C}$ & 0.000 & $\mathrm{C}$ & 0.110 & $\mathrm{x}-\mathrm{C}$ & 0.000 & $\mathrm{C}$ & 0.038 & $\mathrm{mn}$ & 0.000 \\
\hline $94 f$ & 0.478 & $\mathrm{y}-\mathrm{C}$ & 0.150 & $\mathrm{v}-\mathrm{C}$ & 0.300 & $q-A$ & 0.055 & $\mathrm{z}-\mathrm{C}$ & 0.247 & hijk & 11.506 \\
\hline Jelly & 0.193 & $\mathrm{u}-\mathrm{C}$ & 0.020 & $\mathrm{BC}$ & 0.050 & $\mathrm{z}-\mathrm{C}$ & 0.014 & $\mathrm{C}$ & 0.068 & $\operatorname{lmn}$ & 7.215 \\
\hline Presto & 0.577 & h-n & 0.830 & $d-h$ & 1.480 & B & 0.049 & $\mathrm{z}-\mathrm{C}$ & 0.733 & bc & 8.433 \\
\hline Marabel & 0.125 & $\mathrm{w}-\mathrm{C}$ & 0.300 & $\mathrm{o}-\mathrm{Z}$ & 0.040 & $\mathrm{z}-\mathrm{C}$ & 0.178 & $\mathrm{v}-\mathrm{C}$ & 0.162 & $\mathrm{klm}$ & 142.693 \\
\hline $97 f-267$ & 0.008 & $\mathrm{C}$ & 0.010 & $\mathrm{C}$ & 0.010 & $\mathrm{C}$ & 0.004 & $\mathrm{C}$ & 0.006 & $\mathrm{n}$ & 51.807 \\
\hline Picasso & 0.033 & $\mathrm{z}-\mathrm{C}$ & 0.020 & $\mathrm{BC}$ & 0.030 & $\mathrm{BC}$ & 0.000 & $\mathrm{C}$ & 0.020 & $\mathrm{n}$ & 0.000 \\
\hline proventa & 0.534 & i-r & 0.960 & de & 0.290 & q-B & 0.137 & $\mathrm{v}-\mathrm{C}$ & 0.478 & fg & 25.587 \\
\hline Arinda & 0.705 & e-k & 0.070 & $\mathrm{y}-\mathrm{C}$ & 0.030 & $\mathrm{BC}$ & 0.032 & $\mathrm{z}-\mathrm{C}$ & 0.208 & ijk & 4.496 \\
\hline Bolista & 0.139 & $\mathrm{v}-\mathrm{C}$ & 0.570 & h-o & 0.190 & $\mathrm{u}-\mathrm{C}$ & 0.009 & $\mathrm{C}$ & 0.229 & hijk & 6.715 \\
\hline Sante & 0.065 & $\mathrm{y}-\mathrm{C}$ & 0.080 & $\mathrm{y}-\mathrm{C}$ & 0.010 & $\mathrm{C}$ & 0.000 & $\mathrm{C}$ & 0.039 & $\mathrm{mn}$ & 0.000 \\
\hline Mean Agar & 0.519 & a & 0.432 & $\mathrm{~b}$ & 0.284 & $\mathrm{c}$ & 0.072 & $\mathrm{~d}$ & & & \\
\hline
\end{tabular}

* Means with the same letters are not significantly different at $\mathrm{p} \leq 5 \%$.

** Value for each cv. at $10.0 \mathrm{~g} / \mathrm{l}$ agar as relative to the control.

d. Photosynthetic (PS) pigment contents as affected by agar, genotypes and their interaction:

\section{d.1. Main effect of agar induced water stress:}

Results shown in Table (5) indicate that high agar concentration significantly affected all photosynthetic pigment accumulation. Normal (7.0g/l) agar in the tissue culture medium was compared to high level $(10.0 \mathrm{~g} / \mathrm{l})$. As average over the 7 examined genotypes, results indicated significant decline in chl. a, chl. b and total $(\mathrm{a}+\mathrm{b}) \mathrm{chl}$ and carotenoids under high concentration of agar. As \% of control, chl. a, chl. b, total chl and carotenoids contents were $79.6 \%, 73.4 \%, 77 \%$, and $86 \%$ of control, respectively.

\section{d.2. Main effect of genotypes:}

Potato genotypes tested over the two levels of agar indicated significant differences in their pigment contents (Table 5). Chlorophyll a, b and total chl. as well as carotenoids contents were higher in cv. Bolista compared to the rest of genotypes, while cv. Nicola recorded the least accumulation of photosynthetic pigments. 


\section{d. 3. Effect of agar $x$ genotype interaction}

Agar $\mathrm{x}$ genotype interactions were found significant for the contents of photosynthetic pigments (Table 5). The accumulation of chl. a at high agar level was significantly less than the control for all potato genotypes, except in cv. Nicola which had no change in chl. a under agar-induced water stress. The trend of reduction in chl. a was different among the different cvs. At high agar level $(10.0 \mathrm{~g} / \mathrm{l})$, chl. a contents as $\%$ of control were $74.2,82.3,63.0,88.0,86.4$ and $72.4 \%$ for the cvs. Safran, Universa, Triomph, Diamant, Agria and Bolista, respectively. The contents of chl. b were always lower than the control in all examined potato genotypes, except cv. Nicola. Thus, total chl. had the same trend and represented $77 \%$ of control.

Carotenoids were also, and significantly lower under high agar level than the control in all cvs. However, the degrees of reductions were different among the different cvs. As percent of control, carotenoids were 84, 74.8,
$62.6,95.7,81.0$, and $80.8 \%$ of control in cvs. Safran, Universa, Triomph, Daimant,Agria, and Bolista respectively. Results indicated increase in carotenoids contents under water stress in cv. Nicola as opposite to the rest of genotypes.

\section{e. Effect of agar and genotypes on some biochemical contents:}

\section{e. 1. Main effect of agar:}

In this analysis, free amino acids and proline contents, as well as the activities of antioxidant enzymes (SOD and CAT) in the plantlet tissues were studied in relation to the water stress induced by high agar level in the medium. Results revealed that high agar $(10 \mathrm{~g} / \mathrm{l})$ significantly increased the average free amino acids and CAT activity as an average over the tested genotypes, however, SOD was significantly reduced and proline content was unaffected (Table 6).

Table (5). Effect of in vitro agar- induced water stress on chlorophyll and carotenoid contents in 7 potato cultivars.

\begin{tabular}{|c|c|c|c|c|c|c|c|c|}
\hline \multirow{3}{*}{$\begin{array}{l}\text { CV } \\
\text { Safran }\end{array}$} & \multirow{2}{*}{$\frac{\text { Agar }}{7 g \backslash 1}$} & \multirow{2}{*}{$\frac{\text { Chl. a }}{35.15^{*}}$} & \multirow[b]{2}{*}{$\mathrm{b}$} & \multirow{2}{*}{$\begin{array}{l}\text { Chl. b } \\
21.12\end{array}$} & \multirow[b]{2}{*}{$\mathrm{e}$} & \multirow{2}{*}{$\begin{array}{l}\text { Chl. Total } \\
56.26\end{array}$} & \multicolumn{2}{|c|}{ Carotenoids } \\
\hline & & & & & & & 13.37 & $\mathrm{f}$ \\
\hline & $10 \mathrm{~g} \backslash 1$ & 26.07 & $\mathrm{~h}$ & 15.05 & $\mathrm{k}$ & 41.12 & 11.24 & $\mathrm{i}$ \\
\hline \multirow{4}{*}{ Universa } & Mean CV & 30.61 & $\mathrm{c}$ & 18.08 & $\mathrm{~d}$ & 48.69 & 12.31 & $\mathrm{e}$ \\
\hline & $7 g \backslash 1$ & 29.84 & $\mathrm{e}$ & 23.36 & $\mathrm{~d}$ & 53.19 & 16.51 & $\mathrm{c}$ \\
\hline & $10 \mathrm{~g} \backslash 1$ & 24.56 & $\mathrm{j}$ & 11.32 & $\mathrm{~m}$ & 35.88 & 12.35 & $\mathrm{~g}$ \\
\hline & Mean CV & 27.20 & $\mathrm{~d}$ & 17.34 & $\mathrm{e}$ & 44.53 & 14.43 & $\mathrm{~b}$ \\
\hline \multirow{3}{*}{ Triomph } & $7 g \backslash 1$ & 29.84 & $\mathrm{e}$ & 23.36 & $\mathrm{~d}$ & 53.19 & 16.51 & $\mathrm{c}$ \\
\hline & $10 \mathrm{~g} \backslash 1$ & 18.80 & $\mathrm{~m}$ & 16.69 & $\mathrm{~g}$ & 35.48 & 10.34 & $\mathrm{k}$ \\
\hline & Mean CV & 24.32 & $\mathrm{e}$ & 20.03 & $\mathrm{~b}$ & 44.35 & 13.43 & $\mathrm{c}$ \\
\hline \multirow{3}{*}{ Nicola } & $7 g \backslash 1$ & 21.34 & 1 & 15.95 & $\mathrm{i}$ & 37.27 & 9.39 & 1 \\
\hline & $10 \mathrm{~g} \backslash 1$ & 21.56 & $\mathrm{k}$ & 16.49 & $\mathrm{~h}$ & 38.05 & 14.65 & $\mathrm{~d}$ \\
\hline & Mean CV & 21.45 & $\mathrm{f}$ & 16.22 & $\mathrm{~g}$ & 37.67 & 12.02 & $\mathrm{f}$ \\
\hline \multirow{3}{*}{ Diamant } & $7 g \backslash 1$ & 28.91 & $\mathrm{i}$ & 19.23 & $\mathrm{f}$ & 48.12 & 11.45 & $\mathrm{~h}$ \\
\hline & $10 \mathrm{~g} \backslash 1$ & 25.46 & $\mathrm{f}$ & 14.89 & 1 & 40.35 & 10.96 & $\mathrm{j}$ \\
\hline & Mean CV & 27.18 & $\mathrm{~d}$ & 17.06 & $\mathrm{f}$ & 44.24 & 11.20 & $\mathrm{~g}$ \\
\hline \multirow{3}{*}{ Agria } & $7 g \backslash 1$ & 33.27 & $\mathrm{c}$ & 23.47 & $\mathrm{c}$ & 56.72 & 14.12 & $\mathrm{e}$ \\
\hline & $10 \mathrm{~g} \backslash 1$ & 28.75 & $\mathrm{~g}$ & 15.59 & $\mathrm{j}$ & 44.34 & 11.44 & $\mathrm{~h}$ \\
\hline & Mean CV & 31.01 & $\mathrm{~b}$ & 19.53 & $\mathrm{c}$ & 50.54 & 12.78 & $\mathrm{~d}$ \\
\hline \multirow{3}{*}{ Bolista } & $7 g \backslash 1$ & 41.58 & $\mathrm{a}$ & 35.99 & $\mathrm{a}$ & 77.55 & 20.66 & $\mathrm{a}$ \\
\hline & $10 \mathrm{~g} \backslash 1$ & 30.09 & $\mathrm{~d}$ & 29.22 & $\mathrm{~b}$ & 59.31 & 16.70 & $\mathrm{~b}$ \\
\hline & Mean CV & 35.84 & $\mathrm{a}$ & 32.60 & $\mathrm{a}$ & 68.44 & 18.68 & $\mathrm{a}$ \\
\hline \multicolumn{2}{|c|}{ Mean agar at $7.0 \mathrm{~g} \backslash \mathrm{l}$} & 31.42 & $\mathrm{a}$ & 23.21 & $\mathrm{a}$ & 54.63 & 14.57 & $\mathrm{a}$ \\
\hline \multicolumn{2}{|c|}{ Mean agar at $10.0 \mathrm{~g} \backslash \mathrm{I}$} & 25.04 & $\mathrm{~b}$ & 17.04 & $\mathrm{~b}$ & 42.08 & 12.53 & $\mathrm{~b}$ \\
\hline
\end{tabular}

\footnotetext{
* Means with the same letters are not significantly different at $\mathrm{p} \leq 5 \%$.
} 


\section{e. 2. Main effect of genotypes:}

Averaged over the two agar levels, differences among potato genotypes were detected in their contents of free amino acids, proline, SOD and CAT (Table 6). With respect to total free amino acids, the cvs. Bolista and Diamant (drought sensitive) had significantly higher total free amino acids, followed by cv. Agria, while the cvs. Nicola, followed by Triomph, Universa and Safran had the least free amino acids. Proline contents were also the highest in cvs. Bolista, followed by Agria and Nicola, while the least proline accumulation was recorded in cvs. Safran and Universa, followed by Triomph and Diamant. However, SOD activity was higher in cv. Safran followed by cv. Universa (drought tolerant), and the least activities were found in the tissues of cvs. Bolista and Diamant. The activity of CAT enzyme followed the same trend of free amino acids and proline, with the cv. Bolista having the highest CAT, followed by cvs. Agria and Diamant. The least CAT activity was detected in cv. Nicola followed by cv. Safran (Table 6).

\section{e. 3.Effect of agar $x$ genotype:}

Results indicated that potato genotypes included in this analysis had different responses to the water stress induced by high agar level in the medium. Regarding the contents of free amino acids, the cvs. Safran, Universa, Triomph and Diamant had increased free amino acids contents under $10.0 \mathrm{~g} / \mathrm{l}$ agar as compared to the control, while cvs. Nicola and Agria had significantly less free amino acids at the highest level of agar (Table 6).

With respect to proline contents, the cvs.Safran, Triomph, Diamant and Agria accumulated higher proline at high agar level, while the other cvs. (Universa and Bolista) had lower proline content under water stress imposed by high agar concentration in the medium. In cv. Nicola, the proline content at high level of agar was not significantly different than at the control. The activity of SOD was increased at high agar level in cv. Diamant, while all other cvs had less SOD at higher agar level, compared to control. The activity of CAT showed almost similar pattern to proline. The cvsSafran, Universa, Triomph, Diamant and Bolista, respectively, showed higher CAT activities under high agar-induced water stress, while the reverse was true in cv. Agria. The cv. Nicola had the activity of CAT at high agar $(10.0 \mathrm{~g} / \mathrm{l})$ similar to the control $(7.0 \mathrm{~g} / \mathrm{l})$.

Table (6). Free amino acids, proline and antioxidant profile analysis of seven potato cultivars under high level of agar in vitro

\begin{tabular}{|c|c|c|c|c|c|c|c|c|c|}
\hline \multirow{3}{*}{$\begin{array}{l}\text { CV } \\
\text { Safran }\end{array}$} & \multirow{2}{*}{$\begin{array}{l}\text { Agar } g \backslash \mathbf{l} \\
7 \mathrm{~g} \backslash 1\end{array}$} & \multicolumn{2}{|c|}{$\begin{array}{c}\text { Free amino acids } \\
\mathrm{mg} / \mathrm{g} \text { FW }\end{array}$} & \multicolumn{2}{|c|}{$\begin{array}{l}\text { Proline } \\
\text { mg } \backslash \text { g FW }\end{array}$} & \multicolumn{2}{|c|}{$\begin{array}{l}\text { Superoxide dismutase } \\
\text { (u/gm fresh weight) }\end{array}$} & \multicolumn{2}{|c|}{$\begin{array}{c}\text { Catalase } \\
\text { (u/gm fresh } \\
\text { weight) }\end{array}$} \\
\hline & & $909.00^{*}$ & $\mathrm{gh}$ & 30.870 & fgh & 6947.00 & $\mathrm{a}$ & 1.370 & $\mathrm{~g}$ \\
\hline & $10 \mathrm{~g} \backslash 1$ & 2629.67 & e & 42.330 & efg & 6493.00 & $\mathrm{~b}$ & 4.360 & $\mathrm{f}$ \\
\hline \multirow{3}{*}{ Universa } & Mean CV & 1769.34 & $\mathrm{~d}$ & 36.600 & $\mathrm{~d}$ & 6720.00 & $\mathrm{a}$ & 2.860 & $\mathrm{e}$ \\
\hline & $7 g \backslash 1$ & 772.67 & $\mathrm{~h}$ & 39.200 & efg & 6633.00 & $\mathrm{~b}$ & 1.180 & $\mathrm{~g}$ \\
\hline & $10 \mathrm{~g} \backslash 1$ & 1062.99 & g & 34.370 & $\mathrm{i}$ & 6434.33 & $\mathrm{~b}$ & 7.030 & ef \\
\hline \multirow{4}{*}{ Triomph } & Mean CV & 917.83 & $\mathrm{f}$ & 36.790 & $\mathrm{~d}$ & 6533.67 & $\mathrm{~b}$ & 4.110 & $\mathrm{~d}$ \\
\hline & $7 g \backslash 1$ & 624.00 & $\mathrm{i}$ & 26.630 & ghi & 6657.33 & $\mathrm{~b}$ & 1.140 & $\mathrm{~g}$ \\
\hline & $10 \mathrm{~g} \backslash 1$ & 3302.33 & d & 52.500 & ef & 6093.67 & $\mathrm{c}$ & 7.150 & $\mathrm{~d}$ \\
\hline & Mean CV & 1963.17 & $\mathrm{c}$ & 39.570 & $\mathrm{~cd}$ & 6375.50 & $\mathrm{c}$ & 4.140 & $\mathrm{~d}$ \\
\hline \multirow{2}{*}{ Nicola } & $7 g \backslash 1$ & 2596.00 & e & 66.670 & e & 5983.00 & $\mathrm{c}$ & 1.510 & $\mathrm{~g}$ \\
\hline & $10 \mathrm{~g} \backslash 1$ & 137.33 & $\mathrm{j}$ & 67.500 & e & 4737.00 & $\mathrm{e}$ & 1.190 & $\mathrm{~g}$ \\
\hline \multirow{4}{*}{ Diament } & Mean CV & 1366.67 & e & 67.080 & $\mathrm{c}$ & 5360.00 & $\mathrm{c}$ & 1.350 & $\mathrm{f}$ \\
\hline & $7 g \backslash 1$ & 3507.67 & $\mathrm{c}$ & 34.970 & fg & 5617.00 & $\mathrm{~d}$ & 2.010 & $\mathrm{~g}$ \\
\hline & $10 \mathrm{~g} \backslash 1$ & 4226.33 & $\mathrm{a}$ & 42.670 & efg & 5972.33 & $\mathrm{c}$ & 8.390 & $\mathrm{~d}$ \\
\hline & Mean CV & 3867.00 & $a b$ & 38.820 & $\mathrm{~cd}$ & 5794.67 & d & 5.200 & $\mathrm{c}$ \\
\hline \multirow{2}{*}{ Agria } & $7 g \backslash 1$ & 3870.33 & $\mathrm{bc}$ & 122.670 & d & 6610.00 & $\mathrm{~b}$ & 13.520 & $\mathrm{c}$ \\
\hline & $10 \mathrm{~g} \backslash 1$ & 1482.67 & $\mathrm{f}$ & 139.330 & $\mathrm{c}$ & 6126.67 & $\mathrm{c}$ & 7.970 & $\mathrm{~d}$ \\
\hline \multirow{4}{*}{ Bolista } & Mean CV & 2676.50 & $\mathrm{~b}$ & 131.000 & $\mathrm{~b}$ & 6368.33 & $\mathrm{c}$ & 10.705 & $\mathrm{~b}$ \\
\hline & $7 g \backslash 1$ & 3932.33 & $\mathrm{bc}$ & 197.330 & $\mathrm{a}$ & 4645.33 & $\mathrm{e}$ & 17.290 & $\mathrm{~b}$ \\
\hline & $10 \mathrm{~g} \backslash 1$ & 4008.33 & $\mathrm{~b}$ & 170.670 & $\mathrm{~b}$ & 6427.67 & $\mathrm{~b}$ & 19.010 & $\mathrm{a}$ \\
\hline & Mean CV & 3970.33 & a & 184.000 & $\mathrm{a}$ & 5536.50 & e & 18.150 & $\mathrm{a}$ \\
\hline \multicolumn{2}{|c|}{ Mean agar at $7.0 \mathrm{~g} \backslash \mathrm{I}$} & 2316.00 & $\mathrm{~b}$ & 74.050 & $\mathrm{a}$ & 6156.10 & $\mathrm{a}$ & 5.430 & $\mathrm{~b}$ \\
\hline \multicolumn{2}{|c|}{ Mean agar at $10.0 \mathrm{~g} \backslash \mathrm{I}$} & 2406.80 & $\mathrm{a}$ & 78.480 & $\mathrm{a}$ & 6040.67 & $\mathrm{~b}$ & 7.870 & $\mathrm{a}$ \\
\hline
\end{tabular}

* Means with the same letters are not significantly different at $\mathrm{p} \leq 5 \% .0$. 


\section{DISCUSSION}

In vitro screening of different potato genotypes for drought stress induced by high level of agar was performed using single node and microtuberization bioassays. Physiological and biochemical changes associated with agar stresses factor were examined in selected genotypes, representing different tolerant groups. Result of this study revealed that potato plantlet grown under high concentration $(10 \mathrm{~g} / \mathrm{l})$ of agar in the medium also showed reduced growth compared to the control $(7.0 \mathrm{~g} / \mathrm{l}$ agar) and the highest percentage of decline was noticed in root number. This large decline in rooting may cause restricted water and nutrients uptake from the medium, leading to the observed decrease in plantlet growth. These results are in harmony with those of Daneshmand et al. (2010), Hassanpanah (2009) in potato, and Azami et al. (2010) in tomato tissue culture under PEG- induced water stress. Using high agar level as water stress agent, Shafqatulla et al. (2007), Gopal et al. (2008) and Nistor et al. (2014) also reported reduction in plantlet height, number of nodes and rooting.

In vitro tuberization of potato was significantly affected by agar-induced water stress. Using agar at $10 \mathrm{~g} / \mathrm{l}$ as water stress factor, microtuber number and the average tuber weight were decreased, reaching $49 \%$ and $7.1 \%$ of the control, respectively. Recent report (Pino et al., 2013) showed that cultivated potato plants are generally sensitive to water stress, had reduced yield and tuber quality.

Under water stress induced by agar, a marked reduction in chlorophyll and carotenoides was observed, in agreement with the results of Daneshmand et al. (2010). The reduction in total chlorophyll was higher in cvs. Universa, Triomph and Bolista than in cvs. Agria, Diamant and Safran, while no decline in chlorophyll content occurred in cv. Nicola (moderately tolerant), indicating that chlorophyll contents were not associated with water stress tolerance in the examined potato genotype.

Biochemical analysis of potato plantlet under water stress indicated that amino acids contents were generally higher under water stress in tolerant classified genotypes (cvs. Safran and Universa) in addition to $\mathrm{cv}$. Triomph, but decreased sharply in moderate tolerant (cv. Nicola) and was almost the same, compared to the control, in sensitive (cv. Bolista). Proline showed general increase under water stress in tolerant (cv. Safran), moderate (cvs. Triomph, Nicola and Agria) and sensitive (cv. Diamant) but decreased under water stress in cvs. Universa and Bolista. SOD decreased in all cvs. under water stress, except cv. Diamant and Bolista (drought sensitive). However, CAT activities under water stress were higher than that of the control. CAT increased shapely in tolerant and moderate cvs., but decreased in the water stress sensitive ones.

In accordance with these results, Pino et al. (2013) and Heuer and Nadler (1998) reported increase proline contents under water stress in potato plantlet in vitro, similar to the finding of Azami et al. (2010) in tomato, but it is not necessarily be associated with the tolerance mechanism. Free amino acids and proline contents were also found to be higher in drought stress tolerant tomato line (Srivastava et al., 1995).

The increase in antioxidant enzyme activities under water stress, including CAT, SOD, GPOX and peroxidase in water stress tolerant genotypes was previously reported by Daneshmand et al. (2010); Soni et al. (2011) and Yu-Jie et al. (2013). However, the decrease in SOD activity in our experiment was in contrast with the previous report. Perhaps CAT has more protective role against water stress than SOD in potato plantlet under the in vitro condition of our study.

\section{REFERENCES}

Aebi, H. (1984). Catalase in vitro. Methods in Enzymology, 105: 121.

Ahmed, K.U. and M.M. Rashid (1990). Erradication of potato virus $\times(\mathrm{pvx})$ by thermotherapy and meristem tip culture. Bangladesh Journal of Plant Pathology, 5: 65-69.

Arvin, M.J. and D.J. Donnelly (2008). Screening potato cultivars and wild species to abiotic stresses using an electrolyte leakage bioassay. Journal of Agricultural Science Technology, 10: 33-42.

Azami, M.A., M. Torabi and E. Jalili (2010). In vitro response of promising tomato genotypes for tolerance to osmotic stress. African Journal of Biotechnology, 9 (26): 4014-4017.

Daneshmand, F.A., M.K. Javad and K. Manouchehri (2010). Physiological responses to $\mathrm{NaCl}$ stress in three wild species of potato in vitro. Acta Physiologiae Plantarum, 32(1): 91-101.

FAO (2012). Statistical database FAOSTAT. Available on line at: http//Faostate.Fao.Org/site 567/default.Aspxx\#ancor.

Gopal, J. and I. Kazuto (2007). In vitro screening of potato against water-stress mediated through sorbitol and polyethylene glycol. Plant Cell Reports, 26: 693-700.

Gopal, J., K. Iwama and Y. Jitsuyama (2008). Effect of water stress mediated through agar on in vitro growth of potato. In Vitro Cellular \& Developmental Biology Plant, 44: 221-228.

Hassanpanah, D. (2009). In vitro and in vivo screening of potato cultivars against water stress by polyethylene glycol and potassium humate. Biotechnology, 8: 132-137.

Heuer, B. and A. Nadler (1998). Physiological response of potato plants to soil salinity and water deficit. Plant Science, 137(1): 45-51.

Lee, Y.P. and T. Takabashi (1966). An improved colorimetric determination of amino acids with the use of ninhydrin. Analytical Biochemistry, 14: 7177.

Lichtenthaler, H.K. (1987). Chlorophylls and carotenoids: Pigments of photosynthetic biomembranes. Methods in Enzymology, 148: 350-382.

Morpurgo, R. (1991). Correlation between potato clones grown in vivo and in vitro under sodium chloride stress conditions. Plant Breeding, 107: 80-82. 
Murashige, T. and F. Skoog (1962). A revised medium for rapid growth and bioassays with Tobacco tissue culture. Physiologia Plantarum, 15: 473-497.

Ni, X., S.S. Quisenberry, T. Heng-Moss, J. Markwell, G. Sarath, R. Klucas and F. Baxendale (2001). Oxidative responses of resistant and susceptible cereal leaves to symptomatic and nonsymptomatic cereal aphid (Hemiptera: Aphididae) feeding. Journal of Economic Entomology, 94: 743-751.

Nishikimi, M., N.A. Roa and K. Yogi (1972). Measurement of Superoxide dismutase. Biochemical and Biophysical Research Communications, 46: 849-854.

Nistor, A., N. Chira, M. Cioloca, M. Popa and N. Brasov (2014). Effect of simulated hydric stress in vitro over behavior of different potato varities plantles. Studia Universitatis "Vasile Goldiş", Seria Ştiințele Vieții, 24(2):279-282.

Pino, M.T., A. Avila, A. Molina, Z. Jeknic, T.H.H. Chen (2013). Enhanced in vitro drought tolerance of Solanum tuberosum and Solanum commersonii plants overexpressing the ScCBF1 gene. Ciencia e Investigacion Agraria, 40(1):171-184. 38.

Sabbah, S. and T. Moshe (1990). Development of callus and suspension cultures of potato resistant to $\mathrm{NaCl}$ and mannitol and their response to stress. Plant Cell, Tissue and Organ Culture, 21: 119-128.

Sadasivam, S. and A. Manickam (1991). Amino acids and proteins. In biochemical methods for agricultural sciences (Wiley eastern limited and Tamil Nadu agricultural university, Coimbtore). P: 33-95.

Shafqatulla, K., Raziudin and J. Hamidulla (2007). In vitro screening of potato verities to various agar concentrations. Sarhad Journal of. Agriculture, 23(3):611-616.

Singh, G. (1969). A review of soil moisture relationships in potato. American Potato Journal, 46:398-403.

Soni, P., M. Rizwan, K. Bhatt, T. Mohapatra and G. Singh (2011). In-vitro response of Vigna aconitifolia to drought stress induced by PEG 6000. Journal of Stress Physiology \& Biochemistry, 7: 108-121.

Srivastava, D.K., V.K. Gupta and D.R. Sharma (1995). In vitro selection and characterization of water stress tolerant callus cultures of tomato (Lycopersicon esculentum L.). Indian Journal of Plant Physiology, 38: 99-104.

Yuan, B-Z, S. Nishiyama and Y. Kang (2003). Effects of different irrigation regimes on the growth and yield of drip-irrigated potato. Agricultural Water Management, 63: 153-167.

Yu-Jie, C., Xi-P. Deng, Sang-S. Kwak, W. Chen and A.E. Eneji (2013). Tolerant potato cultivar selection under multiple abiotic stresses. Journal of Food, Agriculture and Environment, 11(2): 760766.

\section{مست معملي لعديد من التراكيب الوراثية المختلفة من البطاطس للإجهاد المائي باستخدام مستويات مرتفعة

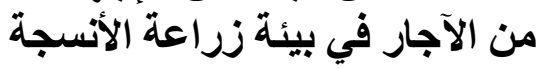 \\ نهلة أحمد المغاورى'، فؤاد حسن محمد'، خالد السيد عبد الحمبدَ، محمد وصفى محمد علوان'، محمد محمد عبد السلام'

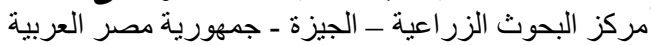

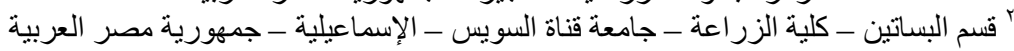

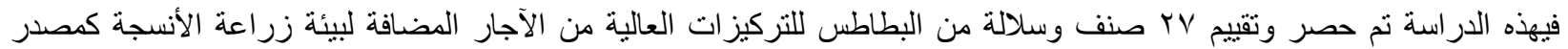

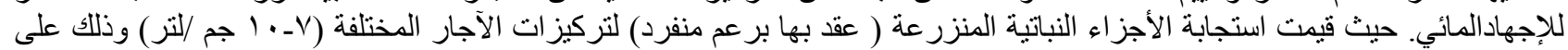

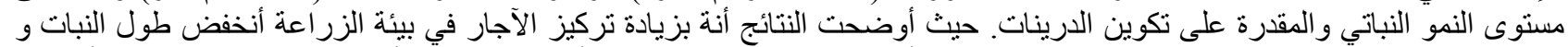

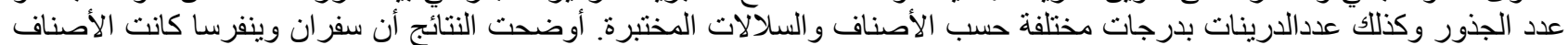

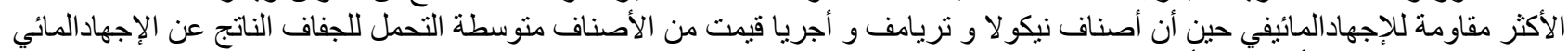

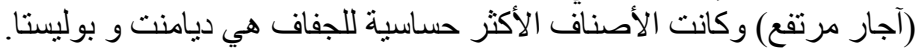

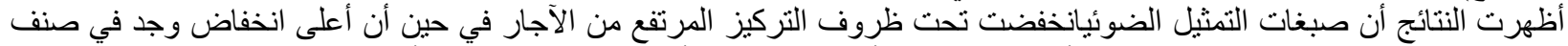

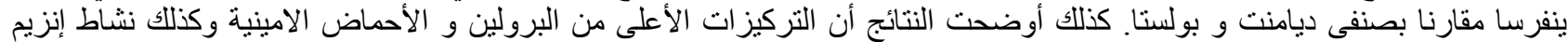

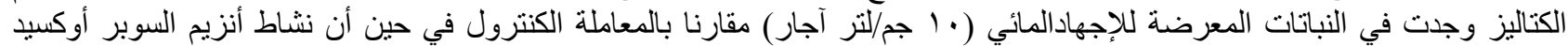

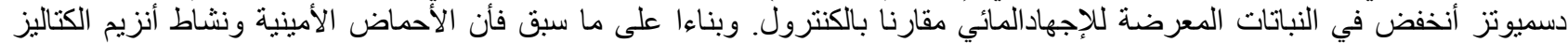
تر افقت ايجابيا مع ميكانيكية مقاومة أصناف البطاطس تحت الدرة لإسة للإجهاد المائي. 